\title{
Resource Allocation and Control Signaling in the WINNER Flexible MAC Concept
}

\author{
Mikael Sternad \\ Signals and Systems, Uppsala \\ University \\ Uppsala, Sweden \\ mikael.sternad@signal.uu.se
}

\author{
Tommy Svensson \\ Dept. of Signals and Systems, \\ Chalmers University of Technology \\ Göteborg, Sweden \\ tommy.svensson@chalmers.se
}

\author{
Martin Döttling \\ Nokia Siemens Networks, \\ St.-Martin-Str. 76, 81541 Munich, \\ Germany \\ martin.doettling@nsn.com
}

\begin{abstract}
The EU WINNER projects have studied OFDM-based packet data systems beyond 3G that use adaptivity on all timescales to obtain high flexibility and performance. The adaptive transmission in both downlink and uplink is scheduled and controlled at base stations and relay nodes and requires frequent transmission of control information over the downlink. The use of scheduling, adaptive modulation and coding, with fine granularity in both time and space, could potentially result in unrealistic bandwidth demands for such downlink control signaling. The present paper describes how this problem has been handled within WINNER in two cases: Frequency-adaptive transmission, which allows individual link adaptation within time-frequency resource units and non-frequency adaptive transmission, which averages over the channel variations in the frequency domain. An important tool for limiting the associated control information is to broadcast only a small essential set of control data to all user terminals, using a safe but therefore bandwidth-demanding code rate. The remaining control information is multicast to groups of users with different signal to interference and noise ratios (SINRs). The modulation and code rates of these transmissions are adjusted to the SINRs of these groups. The over-all coded data rate of the control transmission can thereby be reduced to acceptable levels.
\end{abstract}

Keywords-OFDM;IMT-Advanced; Adaptive transmission; $M A C$; transmission control; control overhead.

\section{INTRODUCTION}

The European research projects WINNER (2004-2005) and WINNER II (2006-2007) have involved more than 40 partners from industry, operators, and academia, partly funded by the European Union. The overall goal has been to develop a single radio interface that covers a range of scenarios from isolated hot spots to wide area cellular deployment, by using different modes of a common technology [1], [2]. The targets are increased data rates, low latency, and high system capacity. The work has resulted in a proposed design and assessment of a beyond $3 \mathrm{G}$ system [2]. The proposed protocol architecture is similar to that of 3GPP LTE [3], to facilitate integration of the proposed concepts into future standardization. Novel elements include adaptive transmission, support for flexible spectrum usage, relaying and advanced time-frequency-spatial adaptive transmission schemes.
As for most proposals for 4G/IMT-Advanced systems, the WINNER radio interface design is based on multicarrier transmission. It is aimed at attaining high flexibility in terms of spectrum use, fulfillment of user requirements and a high spectral efficiency; goals that are often contradictory and difficult to combine. The medium access control (MAC) layer of the protocol architecture plays an important role for fulfilling these goals. It performs resource allocation and scheduling on a short time-scale and thereby controls the packet processing in the physical, MAC and radio link control (RLC) protocol (sub)layers. It controls and optimizes the packet segmentation, encoding, link adaptation and the spatial processing.

The present paper focuses on the allocation of timefrequency resources and the associated downlink control signaling that is required to support this allocation. The adaptive transmission in both downlink and uplink is assumed to be scheduled and controlled by MAC layer schedulers that are physically located in base stations or relay nodes. The resulting allocation decisions are specified via control signaling over the downlink.

The assumed use of scheduling, adaptive modulation and coding with fine granularity in both time and space could potentially result in an unrealistically large control signaling overhead. The problem is complicated by the demand for low transmission latency and high flexibility of the air interface, and the demand for efficient transmission of bursty packet data. This limits the possibility to use fixed resource preallocations, which would require much less control overhead.

We describe how these issues have been handled within WINNER in two cases: Frequency-adaptive transmission which allows individual link adaptation within time-frequency resource units and non-frequency adaptive transmission, which averages over the channel variations in the frequency domain. Please also see the related papers [4] and [5] for a discussion of other MAC layer design aspects and the integration of spatial multi-antenna transmit schemes.

\section{TWO TRANSMISSION PRINCIPLES}

Channel diversity refers to the variability of signal-to 
interference and noise ratios (SINRs) in channels that differ with respect to time, frequency, spatial properties or polarization. Management and utilization of the available diversity is an important task of a resource allocation scheme. In particular, appropriate and efficient use of frequency diversity is important in the WINNER system which is targeted towards bandwidths of 5-100 MHz.

There are two main transmission principles that use the channel diversity in opposite ways [6]: Adaptive transmission uses channel quality information at the transmitter to optimize the multi-user allocation and link adaptation. It strives to utilize the channel variability. Diversity based transmission on the other hand uses coding and interleaving to average over different resource elements to reduce the channel variability. The WINNER design uses both of these principles and applies each of them when appropriate.

\section{A. Frequency adaptive transmission}

Frequency-adaptive transmission utilizes the frequency variability of the channel. Up to moderate vehicular velocities, link adaptation and scheduling can be performed with fine granularity in the time-frequency domain (TDMA/OFDMA) [1], [2]. This provides multi-user scheduling gains for mobile as well as stationary terminals [7]. New methods have been developed in which coding over multiple resource blocks can be combined efficiently with adaptive modulation within each resource block [8]. Efficient channel quality feedback schemes [9] support the design. It has recently been shown in a multicell system simulation context that the use of frequencyadaptive transmission significantly improves the spectral efficiency and the number of satisfied users, as compared to a case where each user can use only a single modulation and code rate within its allocated transmission resources [10].

\section{B. Non-frequency adaptive transmission}

Non-frequency adaptive transmission uses averaging over a set of frequency resources. A code block is interleaved and mapped onto transmission resources within a wide frequency range. The same link adaptation is used within the code block. Non-frequency adaptive transmission is useful as a fallback mode when frequency-adaptive transmission cannot be used due to high terminal velocity, low SINR or in other cases where reliable channel quality feedback is not available (e.g. small packet calls). This transmission mode is also useful for robust transmission of time-critical control information which cannot use retransmission as well as for multicasting to users with largely varying channel conditions.

The WINNER projects have evaluated several alternative ways to utilize the frequency diversity. This has resulted in a proposal to use multi-carrier based schemes that allocate small time-frequency blocks exclusively to users, denoted BEFDMA in downlinks and B-IFDMA in uplinks [2]. The schemes use multiple small blocks that are equidistantly dispersed in frequency to provide large frequency diversity also for small packets. The equidistant block allocation enables DFT precoding to reduce the signal envelope variations, thus improving the terminal power efficiency [11] in the uplink, and it reduces the allocation complexity in both downlinks and uplinks. The transmission can also be concentrated in time to enable energy saving schemes in user terminals, base stations and relay nodes.

\section{Deployment scenarios and Example designs}

Within the WINNER projects, a set of test scenarios were defined that use particular parameterizations of the flexible air interface. Table I illustrates two such scenarios: An FDD system with $2 \times 50 \mathrm{MHz}$ bandwidth, mainly evaluated for suburban base coverage with hexagonal cell deployment, and a TDD system with $100 \mathrm{MHz}$ bandwidth, evaluated for smaller cells in metropolitan areas and indoor systems.

TABLE I. OFDM PARAMETERS

\begin{tabular}{|c|c|c|}
\hline & $\begin{array}{c}\text { Base Coverage } \\
\text { Urban (FDD) }\end{array}$ & $\begin{array}{l}\text { Micro- } \\
\text { cellular }\end{array}$ \\
\hline Subcarrier distance $\Delta f$ & $39062.5 \mathrm{~Hz}$ & $48828.125 \mathrm{~Hz}$ \\
\hline Useful symbol duration $T_{N}$ & $25.6 \mu \mathrm{s}$ & $20.48 \mu \mathrm{s}$ \\
\hline Guard interval $T_{G}$ & $3.2 \mu \mathrm{s}$ & $2.00 \mu \mathrm{s}$ \\
\hline Total symbol duration & $28.8 \mu \mathrm{s}$ & $22.48 \mu \mathrm{s}$ \\
\hline Used subcarriers & $\begin{array}{c}{[-576: 576]} \\
\text { subcarrier } 0 \\
\text { unused }\end{array}$ & $\begin{array}{c}{[-920: 920]} \\
\text { subcarrier } 0 \text { unused }\end{array}$ \\
\hline Signal bandwidth & $2 \times 45 \mathrm{MHz}$ & $89.84 \mathrm{MHz}$ \\
\hline System bandwidth & $2 \times 50 \mathrm{MHz}$ & $100.0 \mathrm{MHz}$ \\
\hline $\begin{array}{l}\text { FFT bandwidth, sampling } \\
\text { rate }\end{array}$ & $80.0 \mathrm{MHz}$ & $100.0 \mathrm{MHz}$ \\
\hline
\end{tabular}

Both these example modes use a frame duration of $0.69 \mathrm{~ms}$ (Table II). In the TDD mode, a frame consists of a downlink transmission slot followed by an uplink slot, separated by a duplex guard interval. In the FDD mode, the frame consists of two slots. ${ }^{1}$ A super-frame consists of a number of frames. The WINNER example designs have used 8 frames per superframe. Within each super-frame, sets of time-frequency resources are assumed to be pre-allocated for use for frequency-adaptive and non-frequency adaptive transmission. This is denoted resource division. Subsets of time-frequency resources within the super-frame may furthermore be allocated for use by relay nodes, or for interference avoidance. This preallocation is denoted resource partitioning. The preallocations can be re-adjusted each super-frame, but they

\footnotetext{
${ }^{1}$ The WINNER concept supports both half-duplex and full duplex FDD terminals. In a cell, one set of half-duplex terminals would receive downlink transmissions in the first slot and transmit in the uplink in the second slot. A second set of terminals could do the opposite, since an FDD base station can be assumed to use full duplex. Full duplex FDD terminals could transmit and receive in both slots, which doubles the maximal data rate.
} 
would typically stay constant over longer intervals.

An important aspect for limiting the control signaling is to use an appropriate time-frequency granularity for these preallocations. The basic resource unit for resource division and resource partitioning has in the WINNER projects been denoted a chunk. It comprises of a set of adjacent subcarriers and subsequent OFDM symbols. In multiple-antenna transmission, there are in general several spatial dimensions (layers) within a chunk, denoted chunk layers. Different layers may be allocated to different flows/users, when using spatialdivision multiple access (SDMA). These aspects are, however, not in focus here. Table II exemplifies the chunk dimensions that have been used in the later part of WINNER II.

Another important aspect for limiting the control signaling is to not signal the complete pre-allocation to all user terminals within each super-frame. When a packet flow is initiated, the terminal is notified of the resource subset that this particular flow may utilize. When pre-allocations change, only the user terminals that are directly affected by the change need to be notified.

TABLE II. FRAME PARAMETERS

\begin{tabular}{|l||c|c|c|}
\hline & $\begin{array}{c}\text { Base Coverage } \\
\text { Urban (FDD) }\end{array}$ & $\begin{array}{c}\text { Micro- } \\
\text { cellular }\end{array}$ & Indoor \\
\hline \hline Overall frame duration & \multicolumn{2}{|c|}{$0.6912 \mathrm{~ms}$} \\
\hline $\begin{array}{l}\text { Number of OFDM symbols } \\
\text { per frame }\end{array}$ & 24 & 30 \\
\hline \hline $\begin{array}{l}\text { Chunk layer dimension in } \\
\text { symbols x subcarriers }\end{array}$ & $12 \times 8=96$ & $15 \times 8=120$ \\
\hline $\begin{array}{l}\text { Number of chunks per frame } \\
\text { in time and frequency } \\
\text { direction }\end{array}$ & $2 \times 144$ & $2 \times 230$ \\
\hline Duplex guard time & $0 \mu \mathrm{s}$ & $2 \times 8.4 \mu \mathrm{s}$ \\
\hline
\end{tabular}

For the assumed deployment scenarios, the OFDM parameters of Table I and the chunk sizes of Table II, the channels are reasonably flat within a chunk. This makes the chunk layer appropriate also for use as a basic resource unit for frequency-adaptive transmission: One single modulation format can be used within the whole chunk, with only a small performance reduction (less than 10\%) due to channel variability within chunks. This reduces the required control overhead. Frequency-adaptive transmission is assumed to allocate a set of chunk layers exclusively to a segment from a packet flow and it should be able to use individual link adaptation within each chunk layer.

To enable an equidistant block allocation for BEFDMA/IFDMA, the chunk layers pre-allocated for nonfrequency adaptive transmission must be spaced equidistantly in frequency. Figure 1 below illustrates such a possible allocation within a time-frequency slot (half-frame) of the
FDD mode. The chunk size is here 8 subcarriers by 12 OFDM symbols, while the non-frequency adaptive allocation works with blocks of 4 subcarriers by 3 OFDM symbols. One or several such blocks per chunk could be allocated to one user.

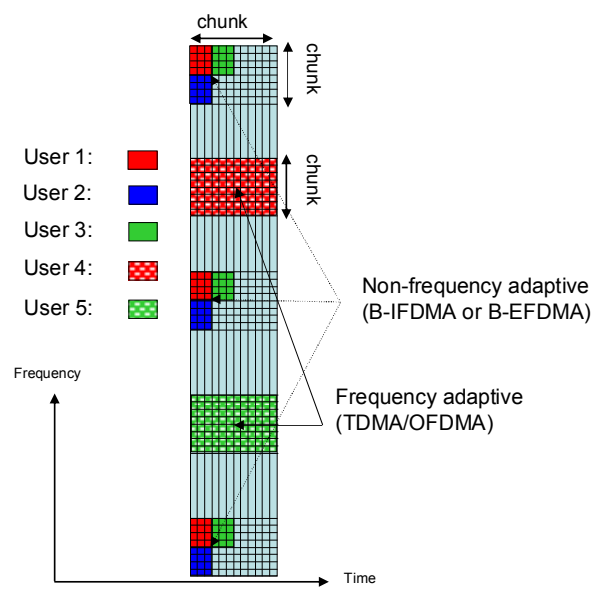

Figure. 1. Time-frequency resource allocation in the frequency-adaptive and non-frequency adaptive transmission modes and the multiplexing of these resource elements in a WINNER FDD slot.

\section{Frame Allocation TABles}

The downlink control information that specifies the destinations and transmission parameters for all downlink and uplink transmission resources within a frame are called frame allocation tables. With demand for scalability from $5 \mathrm{MHz}$ to $100 \mathrm{MHz}$ bandwidth, support of a large variety of service data rates, and a flexible allocation of a large number of resource blocks, the frame allocation tables could require unacceptable overhead. This information is both important and time-critical. Its reliability has to be based on forward error correction, since retransmission cannot be used.

The downlink control signaling scheme proposed in WINNER II allows flexible configuration of the control information in a large range of operating conditions (e.g. ranging from a few high-rate users to many low-rate users, full load, low load). To reduce the control overhead, only a minimal amount of information is broadcast to all users using a safe (but resource-demanding) link adaptation in each frame. More detailed information is transmitted within the same frame to groups of users with differing SINRs, using tailored link adaptation. Below we show examples that illustrate the resulting downlink control overhead for frequency-adaptive and non-frequency adaptive transmission.

\section{A. Non-frequency adaptive transmission}

Consider the allocation of time-frequency blocks to users in the FDD system outlined in Tables I and II. A slot contains 1152 subcarriers $\times 12$ OFDM symbols -576 pilots $=13248$ time-frequency symbols. We make the following assumptions:

1. Individual $4 \times 3$ blocks are allocated to small packets. Large 
packets use multiples of 16 such blocks.

2. The control signaling should work down to SINR $-8 \mathrm{~dB}$.

3. The control signaling uses punctured convolutional codes with base code rate $1 / 4$, combined with repetition coding to reach lower code rates.

4. The allocation tables may use one of $N_{C G}$ modulation and coding schemes (MCS), e.g for $N_{C G}=4$ 4-QAM rate $1 / 2,4-$ QAM rate 1/4 combined with repetition code rates 1, 2 and 6 .

5. We may allocate one, two, four or eight blocks per chunk to one user (B-EFDMA block type). The pattern of all allocated blocks with respect to frequency and space (layer) will be known to the receiver if an initial block location and a total number of blocks are given.

Table III illustrates the information in an entry of the allocation table (AT) that defines the transmission of a code block segment over the air interface without further restrictions on the allocation. Various restrictions on the allocation can reduce the entry size in half to around 20 bits.

TABLE III. EXAMPLE OF ALLOCATION TABLE (AT) ENTRY FOR NONFREQUENCY ADAPTIVE TRANSMISSION.

\begin{tabular}{|l|l|}
\hline Cell specific user address & 9 bits \\
\hline Modulation (2 bits) and coding (3 bits) & 5 bits \\
\hline $\begin{array}{l}\text { Segment size (7 bits) and start position (7 bits) in } \\
\text { terms of blocks for small segments, in multiples of } \\
16 \text { blocks for large segments }\end{array}$ & 14 bits \\
\hline B-EFDMA block type & 2 bits \\
\hline Indication of the utilized spatial transmit scheme & Max. 5 bits \\
\hline $\begin{array}{l}\text { Retransmission process control: Hybrid ARQ } \\
\text { process no (5 bits) + New packet indicator (1 bit) }\end{array}$ & 6 bits \\
\hline Control bits per coded payload segment: & $\mathbf{4 1 ~ b i t s ~}$ \\
\hline
\end{tabular}

The downlink slot needs to contain an allocation table for the following uplink slot as well as for the downlink itself. If these two allocation tables were broadcast to all users, this would require the use of the lowest modulation and code rate (4QAM, rate $1 / 4$, with 6 times repetition coding, resulting in 12 symbols per bit) to reach the worst $-8 \mathrm{~dB}$ terminals with a packet error rate below $0.1 \%$. The resulting downlink overhead would grow with a decreasing average segment size, reaching $50 \%$ for coded and modulated average segment sizes of 1000 symbols and approaching $100 \%$ for smaller average segment sizes.

The overhead can fortunately be reduced significantly by using modulation and code rates that are appropriate for the intended receiver. But the receivers would need information about where within these sets of tables they can find the relevant entry or entries. This problem is solved as follows: A small configuration table (CT) is located at a position known to all receivers and is broadcasted with 12 symbols per bits to all users. It contains the number of allocation table (AT) entries that use each of the four allowed MCSs. By reading the CT, each user terminal will know the location (the offset from the start position) of the AT entries that have been encoded with its relevant MCs. The resulting overhead is low. For example, with 5-bit entries and four MCSs, the configuration tables for uplink and downlink require $2 \times 5 \times 4 \times 12$ symbols $/$ bit $=480$ time-frequency symbols, or $3.6 \%$ overhead per downlink FDD slot.

The overhead due to the allocation table entries with adjusted MCSs will depend on the SINR distribution of users within the cell. In realistic examples, this overhead for downlink plus uplink allocation tables is less than $20 \%$ of the downlink FDD slot, if restricted allocation schemes (20 bit table entries) are used for users with very low SINRs, below $0 \mathrm{~dB}$.

\section{B. Frequency-adptive transmission: Flexible case.}

For frequency-adaptive transmission, individual link adaptation per chunk is used and the resource allocation doesn't follow a regular pattern. Thus, a further refinement of the procedure is required, as depicted in Fig. 2. As the overall length of the control signaling is varying, a length indicator (LI) consisting of a pointer ptr_start to the first data-carrying chunk is inserted after the $\mathrm{CT}$ in order to avoid unused resources.

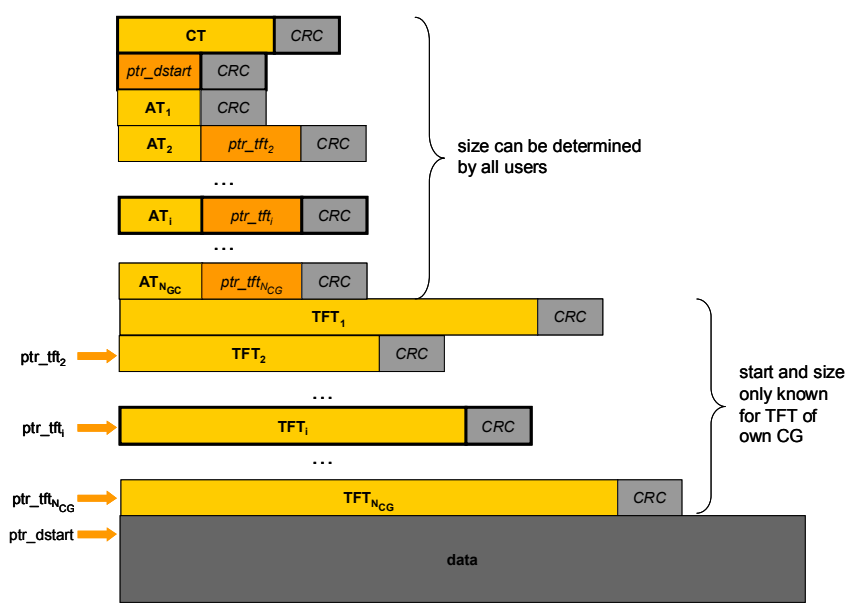

Figure. 2. Adaptive Control Signaling Concept for Frequency-Adaptive Transmission.

To further reduce the overhead, the scheme selects between two basic table construction principles:

- Case A, combinatorial allocation: Resource allocation and transport format information are further separated into AT and Transport Format Table (TFT), transmitted individually to the $N_{C G}$ groups of users. The $\mathrm{AT}$ and $\mathrm{TF}$ together indicate explicitly to each user which chunks are allocated to it, i.e. a sequence of chunk IDs is signaled per user. The overall control message structure is depicted in Fig. 2.

- Case B, table based approach: A sequence of short-hand user IDs is signaled in the TFT, where the position in the sequence implicitly maps to the corresponding chunk ID. The AT does not contain resource mapping information.

The ATs have sizes known to all users and contain pointer $p \_t f t_{i}$ to the corresponding TFT, which have flexible length 
according to the actual resource scheduling decision. The TFT contains all information required for decoding, such as transport block size, MIMO scheme, code rate, adaptive modulation per chunk, and HARQ-related information, see [2] for more details on the adaptive control signaling.

To estimate the control overhead of this approach, the infinite combinations of resource scheduling decisions and user channel conditions need to be restricted. Thus, the following additional main assumptions were used: the CT is updated every $2^{\text {nd }}$ superframe, the LI is sent every slot, and the SINR distribution of the scheduled user results in an average coding efficiency of 2 symbols / control information bit / slot for the AT and TFT. Furthermore it is assumed that an equal number of chunks is allocated to all scheduled users. In order to obtain upper bounds on control overhead, maximum flexibility was assumed in resource scheduling, i.e. all users can be allocated an arbitrary sequence of chunks and four spatial chunk layers need to be signaled with individual modulation.

Fig. 3 shows an example for the WINNER FDD mode with 320 active users (i.e. users that can be scheduled). The $\mathrm{x}$ axis denotes the number of chunks or resource elements (RE) per user, i.e. ranges from scheduling many low rate users to few high-rate users. The overhead contribution of the CT and LI is negligible. If up to 35 chunks are allocated per user the tablebased approach is used. For allocation per user larger than 35 chunks the combinatorial allocation is more beneficial and hence a combination of AT and TFT leads to the minimum control overhead. For any of the investigated cases the total control overhead can be kept below $24 \%$.

Additional investigations in [2] show that control overhead can be kept low for a wide range of usage scenarios, ranging from few active users (e.g. 10\% overhead for 8 active users) to an extremely high number of active users (e.g. around $22 \%$ for 1280 users in TDD mode).

\section{CONCLUSIONS}

The novel WINNER resource allocation and downlink control signaling concept has been explained and illustrated by means of examples. It avoids the need for blind detection and is based on a flexible-length multi-part control message. Total overhead is minimized by adapting the control message format between a combinatorial and table-based approach to signal the resource allocation. Overhead can be kept below $24 \%$ while having full scheduling flexibility for up to 1280 users in a $100 \mathrm{MHz}$ bandwidth system. Further reduction of control overhead can be obtained by restricting the flexibility, e.g. exploiting coherence bandwidth, coherence time, or regular traffic patterns, e.g. by using persistent scheduling techniques [13].

\section{ACKNOWLEDGMENT}

This work has been performed in the framework of the IST project IST-4-027756 WINNER II, which was partly funded by the European Union. The authors would like to acknowledge the contributions of their colleagues in WINNER II, although the views expressed are those of the authors and do not necessarily represent the project.

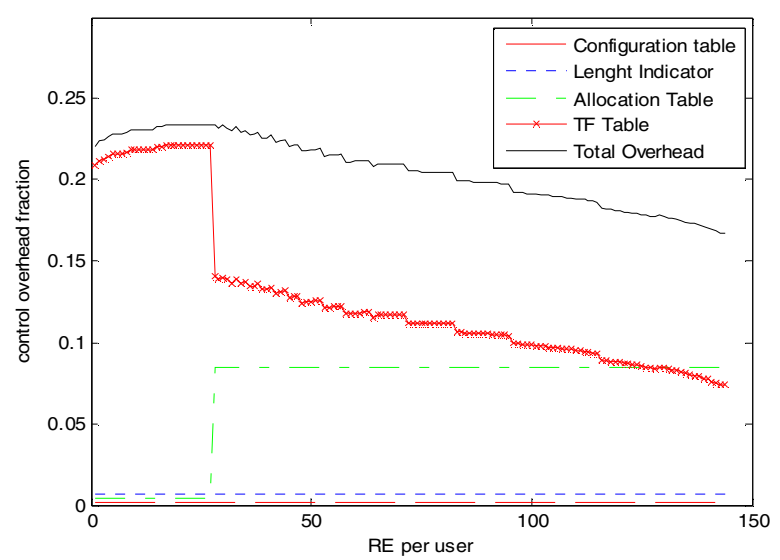

Figure. 3. Overhead Estimation: FDD mode, 320 active users organized in $N_{C G}=5$ control groups: Total downlink control overhead, as functions of the number of resource elements (RE) or chunks allocated per user. This number is in this example assumed equal for all users.

\section{REFERENCES}

[1] IST-2003-507581 WINNER, "D2.10 Final report on identified RI key technologies, system concept, and their assessment”. December 2005. Available at http://www.ist-winner.org.

[2] IST-4-027756 WINNER II, "D6.13.14 WINNER II System Concept Description", November 2007. Available at http://www.ist-winner.org.

[3] 3GPP TS 36.300. Evolved Universal Terrestial Radio Access (E-UTRA) and Evolved Universal Terrestial Radio Access Network (EUTRAN);Overall Description: Stage 2 (Release 8).V 8.0.0, April 2007 .

[4] M Sternad, T. Svensson and G. Klang, "The WINNER B3G MAC Concept," IEEE VTC 2006-Fall, Montreal, Canada, Sept. 2006.

[5] M. Döttling, M. Sternad, G. Klang, J. Von Häfen and M. Olsson, "Integration of spatial processing in the WINNER B3G Air interface design," IEEE VTC 2006-Spring, Melbourne, May 2006.

[6] M. Sternad, T. Svensson, T. Ottosson, A. Ahlén, A. Svensson and A. Brunstrom, "Towards systems beyond 3G based on adaptive OFDMA transmission," Proceedings of the IEEE, vol. 95, Dec. 2007, pp. 24322455.

[7] M. Sternad, S. Falahati, T. Svensson and D. Aronsson, "Adaptive TDMA/OFDMA for wide-area coverage and vehicular velocities," IST Mobile Summit. Dresden, July 2005.

[8] S. Stiglmayr, M. Bossert and E. Costa, "Adaptive coding and modulation in OFDM systems using BICM and rate-compatible punctured codes, "European Wireless, Paris, April 2007,

[9] T.Eriksson and T. Ottosson," "Compression of feedback for adaptive transmission and scheduling," Proceedings of the IEEE, vol. 95, no. 12, Dec. 2007, pp. 2314-2321.

[10] Krystian Safjan, Jakub Oszmiánski, Martin Döttling and Adrian Bohdanowicz, "Frequency-Domain Link Adaptation for Wideband OFDMA Systems," Proc. WCNC 2008, pp. 1703 - 1708, March 31April 3, 2008.

[11] T. Svensson, T. Frank, D. Falconer, M. Sternad, E. Costa and A. Klein, "B-IFDMA - A power efficient multiple access scheme for nonfrequency adaptive transmission," IST Mobile and Wireless Summit, Budapest, July 2007.

[12] IST-4-027756 WINNER II, "D6.13.7 Test scenarios and calibration cases, Issue 2. December 2006.

[13] D. Jiang, H. Wang, E. Malkamaki, and E. Tuomaala, " Principle and Performance of Semi-Persistent Scheduling for VoIP in LTE System," WiCom 2007, Shanghai, China, Sept. 2007. 